Review

\title{
Hering-Breuer Reflex and pre-Botzinger Complex: A Literature Survey
}

\begin{abstract}
Ahmed M
The existence and physiological role of Hering-Breuer reflex and pre-Botzinger complex has long been depreciated by the Bangladesh society of physiologist (personal communication). The aim of this mini review is to highlight the recent findings on the aforementioned topics. Due to the difficulties in vivo studies in human subjects, many aspects of the neuronal regulation of the respiratory rhythm are still unclear. However, the recent localization of the pre-Botzinger complex in humans and advances in technologies necessitates further exploration of the neuronal circuits in the pre-BotC complex which will subsequently unwrap the magical box and pave the way to solve the puzzle of the mechanism of respiratory rhythmogenesis and its modulation in different pathophysiological conditions.
\end{abstract}

Key Words: Physiology; Hering-Breuer reflex; pre-Botzinger complex; Rhythmic respiration

J Bangladesh Soc Physiol. 2007 Dec;(2):89-94.

For author affiliations, see end of text.

http://www.banglajol.info/index.php/JBSP

\section{Introduction}

$\mathbf{J}$ osef Breuer, a clinical student at the department run by Ewald Hering, in 1868 studied on artificial inflation of lungs of cats, dogs and rabbits and found that passive expansion of the lungs reflexly either shortened inspiratory activity ${ }^{1,2}$ or prolonged expiratory activity $^{3}$ depending on the timing of the inflation stimulus within the respiratory cycle (HeringBreuer inflation reflex). Several studies have shown that the effect of lung inflation on respiratory pattern varies with respiratory phase both in vivo and in vitro.

Lung inflation during inspiration terminates inspiratory activity (Hering-Breuer inspiratory reflex); early in expiration or in mid-expiration lengthens expiration (Hering-Breuer expiratory reflex) and late in expiration has no effect on expiratory duration ${ }^{3,4}$. Conversely, preventing inflation, i.e., deflation of lungs tends to initiate inspiratory activity and prolongs inspiratory time (Hering-Breuer deflation reflex) ${ }^{1}$. These reflexes were found to be dependent on the integrity of the vagus nerve as vagotomy abolished the reflex and breathing become slower and deeper, presumably because of the removal of the afferent pathway of the reflex. This minireview will briefly focus on the Hering-Breuer reflex pathway, its neuronal circuits and the physiological importance of this reflex in human beings.

\section{Reflex Pathway}

The Hering-Breuer inflation reflex is mediated by slowly adapting pulmonary mechanoreceptors (SARs) ${ }^{5}$. In experimental animals as the reflexes were obtained with lung pressure changes within the physiological range, it has been suggested that the SARs are likely being activated ${ }^{5-7}$. SARs are inflation sensitive, respond rapidly to changes in lung pressure, but fire tonically in relation to lung volume. In vivo studies revealed that activation of SARs excite neurons in the medial nucleus tractus solitarius (NTS), which project 
to the ventrolateral medulla to excite subsets of expiratory neurons in and rostral to the preBotzinger complex. In turn, inspiratory neurons are inhibited, thereby lengthen expiration ${ }^{4,8}$.

Recently Ezure et $\mathrm{al}^{9}$ have investigated the central pathway of SARs by visualizing the fluorescent labeled projections of relay neurons (P-cells, pump-cells). Stained P-cells are located in solitary tract and all P-cells have axonal branches in the NTS. The axons from $70 \%$ of the P-cells are projected to nucleus ambiguus and its vicinity (ventral or ventrolateral to the nucleus ambiguus) $^{9}$. In rats, the P-cells located in the intermediate subnucleus of the NTS are found to be responsible for Hering-Breuer reflex ${ }^{7}$. The inspiratory-â (Iâ) cells, located in the ventrolateral NTS, also receives an excitatory input from the SARs ${ }^{7}$. In the cat, most Iâ cells have spinal projections that excite the phrenic motorneurons. In short, the targets of SARs inputs include the neurons involved in rhythm generation (e.g., those in pre-Botzinger and Botzinger regions) as well those involved in respiratory pattern formation (e.g., cranial motorneurons and bulbospinal premotor neurons in the ventral respiratory column of the ventrolateral medulla). Whereas the SARs are the receptors for the inflation reflex, the HeringBreuer deflation reflex is mediated via rapidly adapting receptors (RARs) present within the walls of the airway ${ }^{10}$.

\section{Human Studies}

The vast majority of studies of the Hering-Breuer reflex have been performed in anesthetized or decerbrate animals. However, in humans, SARs have been identified histologically in the lungs ${ }^{5}$ and the reflex has also been studied ${ }^{11}$. Electrical stimulation of the vagi in anesthetized humans produced reflex shortening of inspiratory time and bilateral blockade of the vagi abolished this reflex ${ }^{12}$. Unilateral electrical stimulation of the vagi in awake humans elicited a sense of respiratory discomfort but no reflex changes in breathing. Furthermore, expiratory time prolongation consistent with the Hering-Breuer expiratory reflex was observed in anesthetized humans when lungs were inflated during the inspiratory-expiratory transition ${ }^{13}$. Other studies have found evidence of Hering-Breuer inflation reflex activity only at lung volumes well above the normal tidal breathing range during wakefulness and sleep ${ }^{14}$. Polacheck and coworkers ${ }^{15}$ reported that end-expiratory occlusion led to a mean increase in inspiratory timing of $12.5 \%$, in only 3 of 20 healthy but anesthetized subjects showing a $>35 \%$ prolongation. However, electrical stimulation and airway occlusion are potent stimuli and are not typical of eupnic breathing. Therefore, it is generally accepted that the reflexes are largely inactive in adult humans unless the tidal volume exceeds 1 or $1.5 \mathrm{~L}^{12,16}$, as in exercise. In a human study, it was observed that breathing was not inhibited with inflation within tidal range; inhibition just began with inflation volumes of $500 \mathrm{ml}$ and increased as these volumes increased up to 1.2 $\mathrm{L}^{17}$. In contrast to the agreement on the requirement of higher tidal volume for initiation of Hering-Breuer reflex, recent reports reestablish the role of the Hering-Breuer reflex in modulating pulmonary ventilation by controlling the rate and depth of breathing ${ }^{7,12 \text {, }}$ 18-20.

Busha et al ${ }^{12}$ have hypothesized that cortical influences, present during wakefulness, may mask the effects of Hering-Breuer reflex in awake subjects. Similar interpretation has been made by Simon et $\mathrm{al}^{13}$. In one study, the authors employed proportional assisted ventilation in a pseudorandom sequence to unload the respiratory system above and below the perceptual threshold in normal subjects ${ }^{12}$. They found a significant decrease in inspiratory time only during the application of a subthreshold stimulus and conclude that vagally mediated reflexes modulate the timing of respiration on a breath-to-breath basis in awake humans. While studying the occurrence of the Hering-Breuer reflex in adult

J Bangladesh Soc Physiol. 2007 Dec;(2): 89-94. 
humans with normal pulmonary function, Tryfon et $\mathrm{al}^{19}$ have found that the Hering-Breuer inflation reflex is positive in all subjects, whereas the COPD patients were less sensitive to volume changes compared to normal controls. Therefore, it seems that the experimental data regarding the importance of the Hering-Breuer reflex in physiology of respiration depends on the experimental protocol and the state of the subjects. Interestingly, similar to laboratory animals, the Hering-Breuer reflex is strongest in the first few months of life, but, although becoming progressively weaker, it can be stimulated within the tidal breathing range throughout the first year of life ${ }^{14}$. This is supported by the fact that, after birth the term baby tends to have a relatively high mean respiratory rate ( 40.3 breath $/ \mathrm{min}$ ) in the first few days, which rises to 45.1 breath $/ \mathrm{min}$ by the second week, and from then on it begins to fall ${ }^{21}$.

\section{Physiological Importance}

The original concept that the Hering-Breuer reflex is an important determinant of the rate and depth of ventilation is not absolutely convincing. However, the Hering-Breuer inflation reflex is important in the control of breathing in infants. In adults, the inflation reflex may help in minimizing the work of breathing by inhibiting large tidal volumes as well as preventing overdistension of the alveoli at large volumes ${ }^{22}$. The Hering-Breuer deflation reflex probably prevent lung collapse during the periodic sighs that occur normally ${ }^{23}$ and is thought to contribute to the increased ventilation in individuals with a pneumothorax.

The deflation reflex may be important in preventing atelectasis, particularly in individuals who are being mechanically ventilated ${ }^{22,} 23$. The Hering-Breuer deflation reflex is perhaps very important in helping to actively maintain infant's functional residual capacities $(\mathrm{FRCs})^{10}$. It is very unlikely that infant's FRCs are determined passively like those of adults because the inward recoil of their lungs is considerably greater than the outward recoil of their very compliant chest wall ${ }^{16,24}$. The importance of the Hering-Breuer reflex is also supported by the fact that abnormal development of vagal pathways results in breathing disorders during the early postnatal period ${ }^{7}$.

\section{Pre-Botzinger Complex}

Smith and his coworkers have systematically microsectioned the neonatal rat brainstem to precisely localize the regions with neurons critical for respiratory rhythmogenesis. Rhythmically active neurons were found to be confined to a region about 300 micrometers in diameter in the ventrolateral medulla just caudal to the level of retrofacial nucleus and Botzinger complex of expiratory neurons between the nucleus ambiguus and the lateral reticular nucleus ${ }^{25-28}$. This region has been named as pre-Botzinger complex and several studies reported that neural activity in this region is essential for generation and maintenance of rhythmic respiratory activity in neonatal and adult brainstem neurons and motor neurons both in vivo and in vitro ${ }^{27,29-31}$. It should be noted here that schools of different opinion exist as well ${ }^{32}$. Very recently, anatomical localization, cytoarchitecture and functional features of the preBotzinger complex has been described in humans ${ }^{33}$ (Figure 1).

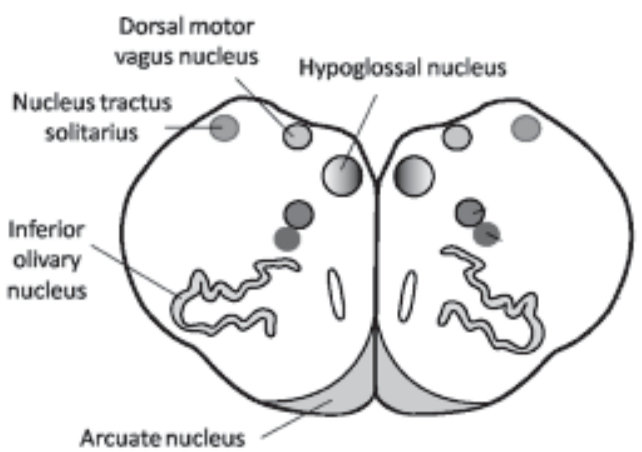

Figure 1. Schematic presentation of a transverse section of the human medulla showing the localization of the pre-Botzinger complex between the nucleus ambiguus and the dorsal accessory of the inferior olivary nucleus. 


\section{Review}

This kernel for rhythm generation consists of a population of neurons with intrinsic pacemaker properties and the activity of these neurons is synchronized by complex synaptic interconnections. The inspiratory neurons with intrinsic oscillatory bursting properties in the preBotzinger complex, onto which several modulatory systems converge, generate the network rhythm and transmit the rhythmic drive to premotor and motor neurons. The preBotzinger complex contains the highest numbers of propriobulbar interneurons, which have direct connections to the more caudal area containing bulbospinal neurons ${ }^{25}$. The interneurons may represent the substrate for transmission of the locally generated rhythm to the bulbospinal premotorneurons that transmit oscillatory drive to phrenic motorneurons and spinal respiratory motorneurons ${ }^{25,} 34$.

Regulation of neuronal network by lung inflation in pre-Botzinger complex

When lungs were periodically inflated within the physiological range in neonatal rats, it modulated respiratory frequency and bursting pattern of six different classes of pre-Botzinger complex (preBotC) respiratory neurons ${ }^{4}$. In control studies all the six different types of neurons identified by Mellen \& Feldman ${ }^{4}$ show bursting pattern during inspiration except the Pre-I neurons ${ }^{30}$ which are active before and after but hyperpolarized during inspiration. The biphasic neurons are silent in control cycles or fires tonically at a low frequency. It has been proposed that this rhythmogenic network of neurons are modulated and reorganized in response to phasic lung inflation that eventually regulate the respiratory frequency (Figure 2).

Type 1 neurons in pre-BotC are characterized by transient outward current $\left(\mathrm{I}_{\mathrm{A}}\right)$ and are hypothesized to be essential in the respiratory rhythm generation ${ }^{29}$. During respiratory rhythmogenesis these neurons begin to depolarize before inspiratory onset and shows ramp-like depolarization during expiration.

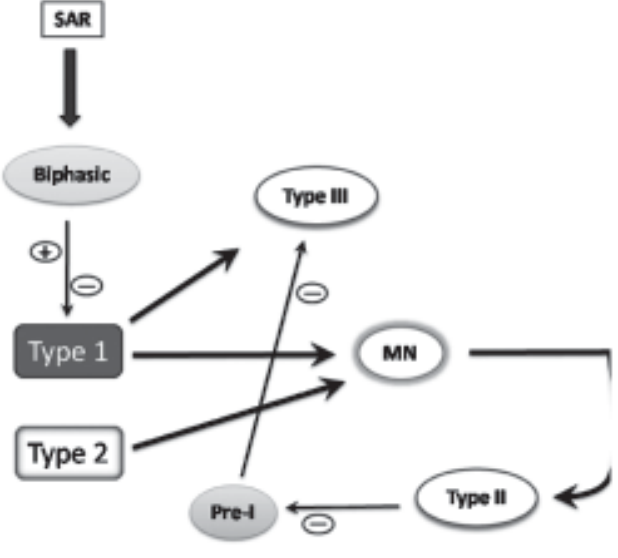

Figure 2. Diagram of potential connectivity between respiratory neurons. SAR afferent input causes rapid firing of biphasic neurons (biphasic) after lung inflation. Postinspiratory inhibition seen only in type 1 neurons (Type 1 ) is consistent with biphasic neuron inhibition of type 1 neurons. Because type 1 inspiratory onset coincides closely with cervical ventral root motor output and fires earliest, type 1 neurons are hypothesized to drive inspiratory premotoneurons, such as type III (Type III) and motoneurons (MN). Delay in onset, together with low cycle-to-cycle variability in burst onset times in type II neurons (Type II), suggests an efferent pathway from motoneurons to type II neurons. Because onset of type II neuron firing precedes but is not significantly different from onset of pre-I inhibition, type II neurons have been hypothesized to inhibit pre-I neurons. Periinspiratory inhibition in type III neurons (Type III) is consistent with inhibition from pre-I neurons; the absence of this inhibition in type II neurons suggests that reciprocal inhibition from pre-I neurons to type II is not present. Because type III firing onset is significantly later than pre-I inhibition, reciprocal inhibition from type III neurons to pre-I neurons is not inferred.

Phasic lung inflations do not change the preinspiratory trajectories but hyperpolarize these neurons and shorten inspiratory firing by $51 \%$. During lung inflation, the biphasic neurons fire rapidly and continued to fire after inflation offset with a decrementing pattern ${ }^{4}$. These neurons are thought to provide GABAergic inhibition of their post-synaptic targets, e.g., type 1 neurons.

J Bangladesh Soc Physiol. 2007 Dec;(2): 89-94. 
Phasic lung inflation induces early onset of preinspiratory depolarization of type 2 neurons in pre-BotC which may contribute to the shortened respiratory cycle. The type II neuron burst onset occurs before pre-I neurons but the preinspiratory hyperpolarization of type III neurons is concurrent with the preinspiratory firing of pre-I neurons. This relative onset times suggest that type II neurons inhibit pre-I neurons which in turn inhibit type III neurons. In phasic lung inflation cycles, the characteristic preinspiratory and postinspiratory firing patterns of pre-I neurons in control cycles are transformed into pure preinspiratory firing. These transformations of firing patterns in subsets of respiratory neurons suggests that in mammals proprioceptive afferent feedback possibly reorganizes central rhythmogenic circuits and play a critical role in generating the respiratory cycle in vivo. However, how the afferent signals modulate the expiratory rhythm and pattern is still unclear. In a recent study, it has been well documented that lung afferent signals excite biphasic and pre-inspiratory neurons in the preBotzinger complex and lengthen the expiration (Hering-Breuer expiratory reflex) ${ }^{35}$. The respiratory neurons in pre-Botzinger complex responsible for the Hering-Breuer reflex have also been characterized in dogs and proposed to play a role in rhythmogenesis ${ }^{36}$.

\section{Author Affiliations}

* Dr Meftun Ahmed, MBBS, PhD

Associate Professor

Department of Physiology

Ibrahim Medical College, Dhaka, Bangladesh \&

Research Fellow

The Oxford Centre for Diabetes, Endocrinology and Metabolism (OCDEM)

Churchill Hospital, University of Oxford

OX3 7LJ, Oxford, UK

Tel: +88 0171542555

Email: meftun.khandker@drl.ox.ac.uk; meftun@hotmail.com

*For correspondence

\section{References}

1. Widdicombe J. Reflexes from the lungs and airways: historical perspective. J Appl Physiol. 2006 Aug;101(2):628-34.

J Bangladesh Soc Physiol. 2007 Dec;(2): 89-94.
2. van Lunteren E. Attenuation of the Hering-Breuer reflex: yet another adverse consequence of COPD? Respiration; international review of thoracic diseases. 2001;68(2):131-2.

3. Mellen NM, Feldman JL. Vagal stimulation induces expiratory lengthening in the in vitro neonate rat. $\mathrm{J}$ Appl Physiol. 1997 Nov;83(5):1607-11.

4. Mellen NM, Feldman JL. Phasic vagal sensory feedback transforms respiratory neuron activity in vitro. J Neurosci. 2001 Sep 15;21(18):7363-71.

5. Schelegle ES. Functional morphology and physiology of slowly adapting pulmonary stretch receptors. The anatomical record. 2003 Jan;270(1):11-6.

6. Mellen NM, Feldman JL. Phasic lung inflation shortens inspiration and respiratory period in the lung-attached neonate rat brain stem spinal cord. Journal of neurophysiology. 2000 May;83(5):3165-8.

7. Kubin L, Alheid GF, Zuperku EJ, McCrimmon DR. Central pathways of pulmonary and lower airway vagal afferents. J Appl Physiol. 2006 Aug;101(2):618-27.

8. Hayashi F, Coles SK, McCrimmon DR. Respiratory neurons mediating the Breuer-Hering reflex prolongation of expiration in rat. J Neurosci. 1996 Oct 15;16(20):6526-36.

9. Ezure K, Tanaka I, Saito Y, Otake K. Axonal projections of pulmonary slowly adapting receptor relay neurons in the rat. The Journal of comparative neurology. 2002 Apr 22;446(1):81-94.

10. Hannam S, Ingram DM, Rabe-Hesketh S, Milner AD. Characterisation of the Hering-Breuer deflation reflex in the human neonate. Respiration physiology. 2001;124(1):51-64.

11. Guz A, Trenchard DW. Pulmonary stretch receptor activity in man: a comparison with dog and cat. The Journal of physiology. 1971 Mar;213(2):329-43.

12. BuSha BF, Judd BG, Manning HL, Simon PM, Searle BC, Daubenspeck JA, et al. Identification of respiratory vagal feedback in awake normal subjects using pseudorandom unloading. J Appl Physiol. 2001 Jun;90(6):2330-40.

13. Simon PM, Zurob AS, Wies WM, Leiter JC, Hubmayr RD. Entrainment of respiration in humans by periodic lung inflations. Effect of state and $\mathrm{CO}_{2}$. American journal of respiratory and critical care medicine. 1999 Sep;160(3):950-60.

14. Hassan A, Gossage J, Ingram D, Lee S, Milner AD. Volume of activation of the Hering-Breuer inflation 
reflex in the newborn infant. J Appl Physiol. 2001 Mar;90(3):763-9.

15. Polacheck J, Strong R, Arens J, Davies C, Metcalf I, Younes M. Phasic vagal influence on inspiratory motor output in anesthetized human subjects. J Appl Physiol. 1980 Oct;49(4):609-19.

16. Rabbette PS, Stocks J. Influence of volume dependency and timing of airway occlusions on the Hering-Breuer reflex in infants. J Appl Physiol. 1998 Dec;85(6):2033-9.

17. Guz A. Hering and Breuer revisited in humans: an invasive study before the days of ethics committees. American journal of respiratory and critical care medicine. 2001 Oct 1;164(7):1110-1.

18. BuSha BF, Stella MH, Manning HL, Leiter JC. Termination of inspiration by phase-dependent respiratory vagal feedback in awake normal humans. J Appl Physiol. 2002 Sep;93(3):903-10.

19. Tryfon S, Kontakiotis T, Mavrofridis E, Patakas D. Hering-Breuer reflex in normal adults and in patients with chronic obstructive pulmonary disease and interstitial fibrosis. Respiration; international review of thoracic diseases. 2001;68(2):140-4.

20. Haberthur C, Guttmann J. Short-term effects of positive end-expiratory pressure on breathing pattern: an interventional study in adult intensive care patients. Critical care (London, England). 2005 Aug;9(4): R407-15.

21. Rabbette PS, Costeloe KL, Stocks J. Persistence of the Hering-Breuer reflex beyond the neonatal period. J Appl Physiol. 1991 Aug;71(2):474-80.

22. Levitzky MG. Pulmonary Physiology. 7 ed: McGrawHill Medical 2007.

23. Cloutier MM. Respiratory Physiology. 1 ed: Mosby 2007.

24. Tepper RS, Williams T, Kisling J, Castile R. Static compliance of the respiratory system in healthy infants. American journal of respiratory and critical care medicine. 2001 Jan;163(1):91-4.

25. Smith JC, Ellenberger HH, Ballanyi K, Richter DW, Feldman JL. Pre-Botzinger complex: a brainstem region that may generate respiratory rhythm in mammals. Science (New York, NY). 1991 Nov 1;254(5032):726-9.
26. Gray PA, Rekling JC, Bocchiaro CM, Feldman JL. Modulation of respiratory frequency by peptidergic input to rhythmogenic neurons in the preBotzinger complex. Science (New York, NY). 1999 Nov 19;286(5444):1566-8.

27. Koshiya N, Smith JC. Neuronal pacemaker for breathing visualized in vitro. Nature. 1999 Jul 22;400(6742):360-3.

28. Johnson SM, Koshiya N, Smith JC. Isolation of the kernel for respiratory rhythm generation in a novel preparation: the pre-Botzinger complex "island". Journal of neurophysiology. 2001 Apr;85(4):1772-6.

29. Rekling JC, Shao XM, Feldman JL. Electrical coupling and excitatory synaptic transmission between rhythmogenic respiratory neurons in the preBotzinger complex. J Neurosci. 2000 Dec 1;20(23):RC113.

30. Onimaru H, Homma I. A novel functional neuron group for respiratory rhythm generation in the ventral medulla. J Neurosci. 2003 Feb 15;23(4):1478-86.

31. Smith JC, Butera RJ, Koshiya N, Del Negro C, Wilson CG, Johnson SM. Respiratory rhythm generation in neonatal and adult mammals: the hybrid pacemakernetwork model. Respiration physiology. 2000 Sep;122(2-3):131-47.

32. St-John WM, Paton JF. Role of pontile mechanisms in the neurogenesis of eupnea. Respiratory physiology \& neurobiology. 2004 Nov 15;143(2-3):321-32.

33. Lavezzi AM, Matturri L. Functional neuroanatomy of the human pre-Botzinger complex with particular reference to sudden unexplained perinatal and infant death. Neuropathology. 2008 Feb;28(1):10-6.

34. Solomon IC. Influence of respiratory network drive on phrenic motor output evoked by activation of cat pre-Botzinger complex. American journal of physiology. 2003 Feb;284(2):R455-66.

35. Mellen NM, Roham M, Feldman JL. Afferent modulation of neonatal rat respiratory rhythm in vitro: cellular and synaptic mechanisms. The Journal of physiology. 2004 May 1;556(Pt 3):859-74.

36. Krolo M, Tonkovic-Capin V, Stucke AG, Stuth EA, Hopp FA, Dean C, et al. Subtype composition and responses of respiratory neurons in the pre-botzinger region to pulmonary afferent inputs in dogs. Journal of neurophysiology. 2005 May;93(5):2674-87.

J Bangladesh Soc Physiol. 2007 Dec;(2): 89-94. 